\title{
FORTALEZAS Y DIFICULTADES DEL COMPORTAMIENTO INFANTIL Y SU RELACIÓN CON LOS FACTORES DE RIESGO SOCIOFAMILIARES
}

\author{
Juan de Dios Uriarte \\ Universidad del País Vasco \\ juandedios.uriarte@ehu.eus \\ Maitane Pegalajar \\ Universidad del País Vasco \\ Itxaso Maniega \\ Universidad del País Vasco
}

Fecha de Recepción: 1 Enero 2019

Fecha de Admisión: 30 Abril 2019

\section{RESUMEN}

El objetivo de este estudio es analizar la relación entre factores de riesgo sociofamiliares y características de comportamiento de niños y niñas del segundo ciclo de Educación Infantil referente a una muestra de 471 alumnos y alumnas y a partir de la información proporcionada por sus maestros/as mediante la versión para profesores del Cuestionario de Capacidades y Dificultades SDQ de Goodman. Los resultados obtenidos indican que los niños y niñas de familias de nivel socioeconómico y/o cultural bajo o muy bajo presentan más problemas de comportamiento y menor conducta prosocial que los alumnos/as de familias de nivel medio 0 alto. La conclusión principal es que hay una relación entre el entorno sociofamiliar y las alteraciones psicológicas y conductuales en el alumnado de preescolar, que pueden ser los antecedentes de otros problemas en edades posteriores.

Palabras claves: riesgo social; SDQ; estatus socioeconómico; problemas psicosociales; educación infantil

\section{ABSTRACT \\ Strengths and difficulties of child behavior and its relationship with sociofamily risk factors. The objective of this study is the analysis of the relationship between social and family risk factors and behavioural characteristics in pre-schoolers. Data have been obtained from a sample of 471 chil- dren, from the information provided by their teachers through the Strengths and Difficulties Questionnaire (SDQ - Goodman). Results indicate that children from families with a low 0 very low socioeconomic and cultural level have more behavioural problems than those from families with a medium o high socioeconomic and cultural level. The main conclusion is that there is a relationship}




\section{FORTALEZAS Y DIFICULTADES DEL COMPORTAMIENTO INFANTIL Y SU RELACIÓN CON LOS FACTORES DE RIESGO SOCIOFAMILIARES}

between the social and family environment and the psychological and behavioural distress of preschoolers, which could be the antecedent of later problems.

social risk, SDQ, socioeconomic status, psychosocial problems, pre-school education

\section{ANTECEDENTES}

A través de las relaciones de apego entre menores y adultos se crean las primeras expectativas de uno mismo y de los demás, que inciden en la autorregulación. Estos modelos internos básicos pueden explicar la relativa continuidad que se observa entre la calidad de las primeras relaciones y las que se establecen en edades posteriores (Ainsworth, 1993; Bolwby, 1980; Crittenden, 1992; Carlson, Sroufe y Egeland, 2004; Thompson, 2000), ya que los problemas sociales, como la exclusión, tienden a reproducirse y transmitirse desde la infancia temprana. Esto lleva a considerar que se debe poner especial cuidado en la influencia de interacciones sociales que ocurren a lo largo del desarrollo infantil.

Durante la infancia, la familia es una de las influencias más importantes en el desarrollo evolutivo y comportamental del niño o niña y por ello, es la encargada de proporcionar el primer y más importante contexto social, emocional, interpersonal, económico y cultural para el desarrollo humano. Las relaciones que se den en su interior tienen una profunda influencia sobre el bienestar infantil; las deterioradas constituyen factores de riesgo comunes y las positivas son factores de protección. Desde esta consideración de la familia, resulta importante conocer las acciones de crianza presentes en los padres y las madres y su relación con el desarrollo de las dimensiones social, emocional, cognitiva, comunicativa, moral y corporal infantiles (Bornstein, Hahn y Haynes, 2011; Lansford, Bornstein, Dodge, Skinner, Putnick y Deater-Deckard, 2011)

La influencia de la clase social es particularmente destacable en las prácticas educativas parentales. Muchos estudios intentan analizar la repercusión de esta en el ambiente del hogar, llegando a la conclusión de que las familias de diferentes clases sociales tratan a los hijos e hijas de modo distinto (McLoyd, Ceballo y Mangelsdorf, 1993). Como resultado, se considera a la clase social como un factor de estudio relevante, ya que es de carácter permanente a lo largo de mucho tiempo.

En el caso del nivel socioeconómico y cultural de la familia, algunos autores mantienen la importancia de un mayor nivel socioeconómico para una mayor calidad en el ambiente familiar (Viguer y Serra, 1996; Barajas y Clemente, 1991), ya que por un lado los niños y niñas en familias pobres tienen un riesgo elevado de padecer problemas conductuales y cognitivos en comparación con los casos procedentes de familias de mayor nivel adquisitivo (Bradley y Corwyn, 2002; Brooks-Gunn y Duncan, 1997; McLoyd, 1998). Y por otro lado, la mayoría de los estímulos que reciben vienen del contexto familiar, por lo que la calidad de estos varía en función de los recursos, capacidades y conocimientos propios del entorno. Según varios autores (Conger \& Donnellan, 2007; Davis, Sawyer, Lo, Priest y Wake, 2010) una de las variables que más afecta al bienestar psicológico de los y las menores es la pertenencia a un nivel socioeconómico bajo, incluso por encima de causas biológicas como pueden ser el nacimiento prematuro o el bajo $\mathrm{Cl}$ (Polou, 2013). En una investigación realizada por Fajardo, León, Felipe, Polo \& Ribeiro (2015), se llegó a la conclusión de que la probabilidad de sufrir problemas de salud y de aparecer comportamientos de riesgo durante la adolescencia se duplica en el caso de menores con familias con nivel socioeconómico y educativo bajo. No obstante, las desventajas que produce un nivel socioeconómico bajo no influyen a todos los niños y niñas por igual, ya que el grado de afección puede verse influido por las diferencias en los factores de riesgo y protectores que se encuentren en su vida diaria (Hartas, 2011).

Sanders (2002), debido a su interés por la prevención, valoró a la familia y a las experiencias ocurrían en su interior, como un punto de partida para iniciar las propuestas de intervención dirigidas a minimizar los problemas de conducta y emocionales infantiles. 
En cuanto al entorno escolar, es donde se puede observar que aunque todos estén escolarizados en la educación infantil, los menores de hogares con más recursos siguen obteniendo mejores resultados. Los procedentes de entornos desfavorecidos es más probable que abandonen antes los estudios y que sigan siendo pobres en edad adulta; la pobreza y la desigualdad se transmiten. Asimismo, algunas investigaciones sugieren que el efecto compensativo entre la Educación infantil y la familia es sistemáticamente mayor entre los niños que viven en familias desfavorecidas. Existen hallazgos en esta dirección referidos a los Estados Unidos (Mc Cartney, Dearing, Taylor y Bub, 2007 y Bassok, 2010), el Reino Unido (Becker, 2011) y Alemania (Felfe y Lalive, 2012).

Desde que el alumnado comienza su recorrido educativo, muestra unas características personales que pueden hacer que se creen unas expectativas más altas o bajas sobre su nivel educativo, lo que conllevará una fuente de satisfacción o de frustración respectivamente. Algunos aspectos familiares, como el ambiente socioeconómico y cultural arriba mencionados, pueden generar situaciones de estrés familiar que el o la menor expresará con ciertas conductas observables.

En la etapa de educación infantil la prevención temprana tiene una gran importancia para evitar problemas en el desarrollo cognitivo y emocional (Clemente, 2011) y para paliar situaciones de desventaja social en la infancia. Teniendo en cuenta el argumento de que el aprendizaje temprano resulta ser el más eficiente (Heckman, 2006; Esping-Andersen, 2008), la educación infantil puede ser decisiva para mitigar la emergencia de desigualdades sociales desde su raíz. Desde la ideología defensora de esta etapa, se argumenta que la inclusión en la escuela infantil de menores provenientes de entorno familiar desfavorecido es la mejor manera de mejorar sus oportunidades vitales a largo plazo.

En esta misma línea, estudios como los de Sveen, Berg-Nielsen, Lydersen \& Wichstrom (2013); Elberling, Linneberg, Olsen, Goodman \& Skovgaard, (2010); y Davis, Sawyer, Lo, Priest \& Wake (2010), reafirman que a pesar de que es un ámbito poco investigado, es de real importancia la investigación de la psicopatología infantil y su intervención temprana, ya que los estudios longitudinales apuntan a la persistencia del trastorno infantil hasta la adolescencia. Por ello, resulta especialmente provechoso comprobar la existencia de una posible relación entre los factores de riesgo social y las características de los comportamientos realizados por el alumnado de Educación Infantil (E.I.).

En el estudio realizado por Briggs-Gowan, Carter, Bosson-Heenan, Guyer \& Horwitz (2006), se indica que los problemas comportamentales y emocionales que aparecen en E.I. no son de carácter transitorio, ya que alrededor de la mitad de los y las afectadas continúan teniendo dichos problemas un año más tarde, agravándose en los casos con trastornos en el desarrollo y/o dificultades familiares.

\section{OBJETIVOS}

Teniendo en cuenta la alta relevancia de la intervención temprana para combatir problemas psicosociales y evitar la futura exclusión social, así como la relación de estos trastornos con el nivel socioeconómico y cultural de la familia, por medio de este estudio se pretende conocer, como objetivo principal, si las desigualdades socioeconómicas y culturales de las familias inciden en el comportamiento del alumnado del segundo ciclo de E.I. Por otro lado, resultaría interesante analizar si estas conductas pueden considerarse un factor de riesgo que actúa sobre las fortalezas y dificultades de estos niños y niñas. Asimismo, se establece una serie de objetivos específicos:

- Describir las fortalezas y dificultades del alumnado de segundo ciclo de E.I., descritas por los maestros y maestras.

- Comparar si la valoración del comportamiento de los niños y niñas por parte de los maestros y maestras es diferente en función de la titularidad del centro (privada-pública). 


\section{MUESTRA}

La muestra completa de este estudio fueron 476 niños $(49,04 \%)$ y niñas $(50,96 \%)$ de segundo ciclo de El (3-5 años $x=4$ años y 11 meses, DT= 0,79). Los colegios elegidos fueron 12, 8 públicos y 4 privados. Las personas informantes fueron 24 tutoras de aula, 17 de colegio público y 7 de privado. El $66,8 \%$ de la muestra total corresponde a colegios públicos y el $33,12 \%$ a privados.

Las familias provenían de diferentes niveles socioeconómicos, según los y las docentes informantes, por los que se categorizaron en cuatro, según su propia percepción: muy bajo (4,67\%), bajo $(36,31 \%)$, medio $(44,80 \%)$ y alto $(14,23 \%)$. Respecto al nivel cultural, se hizo la misma categorización: muy bajo (4,88\%), bajo $(35,03 \%)$, medio $(39,28 \%)$ y alto $(20,81 \%)$. En cuanto a lo que se ha denominado en esta investigación nivel social (n. socioeconómico + n. cultural), fue clasificado como 'Alto' en el $46,8 \%$ de las familias de los alumnos/as de colegios públicos y en el $85,8 \%$ de los colegios privados, y como 'Bajo' en el $53,2 \%$ de los colegios públicos y en el $14,2 \%$ de los colegios privados.

\section{METODOLOGÍA E INSTRUMENTO UTILIZADO}

Para recoger los datos necesarios para este estudio se ha utilizado el Cuestionario de Capacidades y Dificultades -SDQ, Strenghts and Difficulties Questionnaire- (Goodman, 1997), para niños y niñas de $2-4$ años, teniendo en cuenta el comportamiento del niño o niña en los últimos seis meses. Es un instrumento que se utiliza para evaluar el desarrollo infantil y para el cribado de posibles síntomas emocionales y de comportamiento en la infancia y adolescencia. No es un instrumento diagnóstico, sino de detección, que puede alertar sobre problemas emocionales y comportamentales.

Este instrumento está formado por 25 ítems medidos en escala Likert de 3 opciones y mide cinco escalas referidas a: características emocionales, problemas de conducta, hiperactividad, problemas con los compañeros y compañeras y comportamiento prosocial. Tiene una fiabilidad de Alfa de Cronbach en pruebas con preescolares alrededor del 0,8 (Sveen, Berg-Nielsen, Lydersen y Wichstrøm, 2013; Calero, Fernández- Parra, López-Rubio, Carles, Mata, Vives, Navarro \& Márquez, 2013; Tobia, Gabriele \& Marzocchi, 2011). Dependiendo de la puntuación obtenida se puede clasificar al alumnado en tres grupos: Conducta normal para su edad, pequeño riesgo de tener problema en esta área, riesgo importante de tener un problema en esa área.

\section{RESULTADOS}

Para obtener los resultados de esta investigación, se realizó el ANOVA, relacionando por separado cada una de las escalas con las variables del nivel socioeconómico y cultural familiar. Asimismo, la T de Student se utilizó para analizar la relación de cada escala con el nivel social y la posible influencia de la titularidad del colegio en la visión de los maestros y maestras sobre el comportamiento de su alumnado. La distribución de los casos por tipo de colegio según los puntos de corte puede verse en la tabla 1. Se unieron en una sola columna las consideradas como normales y límite, dejando en otro grupo las puntuaciones que muestran un riesgo importante. 
Tabla 1. Distribución de casos por tipo de colegio según los puntos de corte para las escalas del SDQ-T

\begin{tabular}{|c|c|c|}
\hline & \multicolumn{2}{|c|}{$\begin{array}{l}\text { COLEGIOS PUBLICOS } \\
(\mathrm{n}=315)\end{array}$} \\
\hline & $\begin{array}{l}\text { Normal + } \\
\text { limite }\end{array}$ & $\begin{array}{l}\text { Riesgo } \\
\text { importante }\end{array}$ \\
\hline $\begin{array}{l}\text { Sintomas } \\
\text { emocionales }\end{array}$ & $\begin{array}{l}271 \\
(86,0 \%)\end{array}$ & $44(14,0 \%)$ \\
\hline $\begin{array}{l}\text { Problemas de } \\
\text { conducta }\end{array}$ & $\begin{array}{l}283 \\
(89,8 \%)\end{array}$ & $32(10,2 \%)$ \\
\hline $\begin{array}{l}\text { Hiperactivida } \\
\text { d }\end{array}$ & $\begin{array}{l}276 \\
(87,6 \%)\end{array}$ & $39(12,4 \%)$ \\
\hline $\begin{array}{l}\text { Problemas } \\
\text { con los } \\
\text { compañeros y } \\
\text { compañeras }\end{array}$ & 244 & $71(22,5)$ \\
\hline $\begin{array}{l}\text { Dificultades } \\
\text { totales }\end{array}$ & $\begin{array}{l}264 \\
(83,8 \%)\end{array}$ & $51(16,2 \%)$ \\
\hline $\begin{array}{l}\text { Comportamie } \\
\text { nto prosocial }\end{array}$ & $(75,2 \%)$ & $78(24,8 \%)$ \\
\hline
\end{tabular}

\begin{tabular}{|l|l|l|}
\cline { 2 - 3 } \multicolumn{1}{c|}{} & \multicolumn{2}{|c|}{$\begin{array}{c}\text { COLEGIOS PRIVADOS } \\
\text { (n=156) }\end{array}$} \\
\cline { 2 - 3 } \multicolumn{1}{c|}{} & $\begin{array}{l}\text { Normal } \\
\text { limite }\end{array}$ & $\begin{array}{l}\text { Riesgo } \\
\text { importante }\end{array}$ \\
\hline $\begin{array}{l}\text { Sintomas } \\
\text { emocionales }\end{array}$ & $145(92,9 \%)$ & $11(7,1 \%)$ \\
\hline $\begin{array}{l}\text { Problemas de } \\
\text { conducta }\end{array}$ & $147(94,2 \%)$ & $9(5,8 \%)$ \\
\hline $\begin{array}{l}\text { Hiperactivida } \\
\text { d }\end{array}$ & $148(94,9 \%)$ & $8(5,1 \%)$ \\
\hline $\begin{array}{l}\text { Problemas } \\
\text { con los } \\
\text { compañeros } \\
\text { y } \\
\text { compañeras }\end{array}$ & $136(87,2 \%)$ & $20(12,8 \%)$ \\
\hline $\begin{array}{l}\text { Dificultades } \\
\text { totales }\end{array}$ & $144(92,3 \%)$ & $12(7,7 \%)$ \\
\hline $\begin{array}{l}\text { Comportami } \\
\text { ento } \\
\text { prosocial }\end{array}$ & $140(89,7 \%)$ & $16(10,3 \%)$ \\
\hline
\end{tabular}

La escala de síntomas emocionales está formada por cinco ítems y valora las quejas, preocupaciones, infelicidad, dependencia y miedos infantiles. Teniendo en cuenta que la puntuación máxima es 10, la puntuación media obtenida ha sido 1,51 (DT=2,29). Por lo que respecta al nivel socioeconómico percibido, se ha encontrado una diferencia estadísticamente significativa entre los alumnos/as de nivel socioeconómico alto y los de nivel bajo y muy bajo ( $F=13,724$; $S i g=, 000<, 01)$. Asimismo, en cuanto al nivel cultural de las familias percibidas por la tutora, se ha encontrado una diferencia estadísticamente significativa entre el alumnado de nivel socioeconómico alto y el de nivel bajo y muy bajo $(F=7,908 ; S i g=, 000<, 01)$. Al analizar el nivel social, se ha hallado una diferencia estadísticamente significativa entre los niveles alto y bajo $(t=-4,246$; $S i g=, 000<, 01)$. Los y las docentes de los colegios públicos puntuaron los síntomas emocionales infantiles de forma significativamente más alta que los de los privados ( $\mathrm{t}=-3,067$; $\mathrm{Sig}=, 002<, 01)$. El porcentaje de alumnado con una puntuación alta en la escala de Problemas Emocionales, a juicio de sus docentes, fue del $13,70 \%$, de los cuales el $9,21 \%$ estaba escolarizado en un colegio público y el $4,49 \%$ en uno privado.

La escala de Problemas de Conducta está formada por 5 ítems y valora las rabietas, obediencia, peleas, actitud negativa hacia adultos y rencor del alumnado. Siendo la puntuación máxima 10, se ha obtenido una puntuación media de 1,77 (DT=2,35). Por lo que respecta al nivel socioeconómico, se ha encontrado una diferencia estadísticamente significativa entre el alumnado de nivel socioeconómico alto y los de nivel bajo y muy bajo ( $F=9,783$; $S i g=, 000<, 01)$. Asimismo, en cuanto al nivel cultural familiar, se ha encontrado una diferencia estadísticamente significativa entre los menores de nivel socioeconómico alto y los de nivel bajo y muy bajo ( $F=8,567 ; \mathrm{Sig}=, 000<, 01)$. Al analizar el nivel social se ha hallado una diferencia estadísticamente significativa entre los niveles alto y bajo ( $\mathrm{t}=$ $-4,300 ; \mathrm{Sig}=, 000<, 01)$. El porcentaje de alumnado con una puntuación alta en la escala de Problemas de Conducta, a juicio de sus docentes, fue del 15,93\%, de los cuales el 10,16\% estaba escolarizado en un colegio público y el $5,77 \%$ en un colegio privado.

La escala de hiperactividad está formada por 5 ítems y evalúa la inquietud, dificultad de concentración, capacidad para pensar antes de actuar y la tendencia a no terminar las tareas del alumnado. La puntuación máxima es 10 , y la media obtenida es de 3,38 (DT=3,05). Por lo que respecta al nivel socioeconómico, se ha encontrado una diferencia estadísticamente significativa entre los alumnos/as de nivel socioeconómico alto y los de nivel bajo y muy bajo ( $F=23,187$; $S i g=, 000<, 01)$. Asimismo, en cuanto al nivel cultural de las familias, se ha encontrado una diferencia estadística- 
mente significativa entre estudiantes de nivel socioeconómico alto y de nivel bajo y muy bajo $(\mathrm{F}=28,259 ; \mathrm{Sig}=, 000<, 01)$. Al analizar el nivel social se ha hallado una diferencia estadísticamente significativa entre los niveles alto y bajo $(\mathrm{t}=-8,203$; $\mathrm{Sig}=, 000<, 01)$. El profesorado de los colegios públicos puntuaron la hiperactividad de los menores de forma significativamente más alta que los de los privados ( $\mathrm{t}=-3,345 ; \mathrm{Sig}=, 001<, 01$ ). El porcentaje de estudiantes con una puntuación alta en la escala de Hiperactividad, a juicio de sus docentes fue del $17,51 \%$, de los cuales el $12,38 \%$ estaba escolarizado en un colegio público y el $5,13 \%$ en uno privado.

La escala de Problemas con los Compañeros y compañeras está formada por cinco ítems y valora la integración en el grupo, las amistades, el nivel de aceptación y los conflictos con sus iguales. Siendo la puntuación máxima 10, se ha obtenido una media de 1.58 (DT=2,04). Por lo que respecta al nivel socioeconómico, se ha encontrado una diferencia estadísticamente significativa entre alumnado de nivel socioeconómico alto y los de nivel bajo y muy bajo ( $F=13,160 ; S i g=, 000<, 01)$. En cuanto al nivel cultural de las familias, se ha encontrado una diferencia estadísticamente significativa entre estudiantes de nivel socioeconómico alto y los de nivel bajo y muy bajo ( $F=12,906$; $\mathrm{Sig}=, 000<, 01)$. Al analizar el nivel social se ha hallado una diferencia estadísticamente significativa entre los niveles alto y bajo $(\mathrm{t}=-5,216$; $\mathrm{Sig}=, 000<, 01)$. Los docentes de los colegios públicos puntuaron las relaciones de los menores con sus iguales de forma significativamente más alta que los de los colegios privados $(\mathrm{t}=-4,434$; $\mathrm{Sig}=, 000<, 01)$. El porcentaje de alumnado con una puntuación alta en la escala de Problemas con los Compañeros/as, a juicio de sus docentes fue del $35,36 \%$, de los cuales el $22,54 \%$ estaba escolarizado en un colegio público y el $12,82 \%$ en uno privado.

La escala de Comportamiento Prosocial mide la conducta adaptativa. Esta formada, al igual que las anteriores, por 5 ítems y valora la consideración hacia los demás y la disponibilidad para ayudar. La puntuación máxima posible es de 10 puntos y a diferencia de las demás escalas, una puntuación alta es un indicador favorable. La puntuación media es de 6,71 (DT=2,52). Por lo que respecta al nivel socioeconómico, se ha encontrado una diferencia estadísticamente significativa entre el alumnado de nivel socioeconómico alto y los de nivel bajo y muy bajo $(F=13,749 ; \mathrm{Sig}=, 000<, 01)$. Sobre el nivel cultural de la familia, se ha encontrado una diferencia estadísticamente significativa entre los hijos e hijas de nivel socioeconómico alto y los de nivel bajo y muy bajo ( $F=19,658$; $S i g=, 000<, 01)$. Al analizar el nivel social se ha hallado una diferencia estadísticamente significativa entre los niveles alto y bajo ( $\mathrm{t}=-5,839 ; \mathrm{Sig}=, 000<, 01)$. Los maestros y maestras de los colegios públicos puntuaron las relaciones de sus estudiantes con sus iguales de forma significativamente más alta que los de Ios colegios privados ( $\mathrm{t}=-3,802$; $\mathrm{Sig}=, 000<, 01)$. El porcentaje de menores con un Comportamiento Prosocial bajo fue del $35,02 \%$, de los cuales el $24,76 \%$ estaba escolarizado en un colegio público y el $10,26 \%$ en uno privado.

\section{Dificultades Totales}

La escala de Dificultades Totales se obtiene a partir de la suma de las 4 primeras escalas, ya que son las de carácter inadaptativo. La puntuación máxima posible es de 40 puntos y la media de la muestra es de 8,23 (DT=7,85). Por lo que respecta al nivel socioeconómico, se ha encontrado una diferencia estadísticamente significativa entre alumnado de nivel socioeconómico alto y los de nivel bajo y muy bajo ( $F=27,125$; $S i g=, 000<, 01)$. En cuanto al nivel cultural de las familias, se ha encontrado una diferencia estadísticamente significativa entre el alumnado de nivel socioeconómico alto y los de nivel bajo y muy bajo ( $F=26,123 ; S i g=, 000<, 01)$. Al analizar el nivel social se ha hallado una diferencia estadísticamente significativa entre los niveles alto y bajo $(t=-7,759 ; \mathrm{Sig}=, 000<, 01)$. Al comparar las puntuaciones obtenidas en la escala de dificultades totales se ha hallado una puntuación significativamente más alta por parte de docentes de los colegios públicos que por los de los 
colegios privados ( $\mathrm{t}=-4,024 ; \mathrm{Sig}=, 000<, 01)$. El porcentaje de menores con una puntuación alta en la escala de Dificultades Totales fue del $23,88 \%$, de los cuales el $16,19 \%$ estaba escolarizado en un colegio público y el $7,69 \%$ en uno privado.

\section{DISCUSIÓN Y CONCLUSIONES}

Los resultados obtenidos en esta investigación muestran que, según los y las docentes, los niños y niñas de clase social baja y muy baja presentan mayores problemas de comportamiento y tienen menor comportamiento prosocial que los de clase social alta. Estos resultados van acorde a otros estudios (Rodríguez, 2009; Rodríguez-Hernández y Barrau-Alonso, 2012), donde se resalta la importancia de las variables familiares positivas para evitar los problemas de comportamiento en los diferentes contextos de interacción en los que participan los y las menores.

En lo que respecta al tipo de colegio, se han encontrado mayores indicadores de problemas de comportamiento en los colegios públicos; aproximadamente el doble que en los colegios privados. Estos resultados discrepan con otros estudios realizados en España, donde se expone que ciertos tipos de agresiones son menos frecuentes en los centros públicos que en los privados (Defensor del Pueblo, 2007). Asimismo, otra investigación realizada también en España evidencia que, respecto a bullying escolar, hay más agresores en colegios privados que en públicos (León, Felipe, Gómez y López, 2011).

Cabe la posibilidad de que si los problemas de comportamiento que se dan en la etapa de educación infantil, no se solucionan o corrigen, pueden repercutir en las posteriores etapas educativas, dificultando su aprendizaje, y a largo plazo su integración social. En el estudio realizado por Jalón y Arias (2006) se refleja la posibilidad de detectar en la educación preescolar los problemas de exclusión e inadaptación que se manifiesta en la conducta del menor y es observada por el o la docente de aula. Si estos resultados se mantuviesen a lo largo del tiempo, estos niños y niñas formarían familias de bajo nivel socioeconómico y cultural en las que sus propios hijos e hijas podrían presentar los mismos problemas que presentan, posibilitando así la reproducción de las situaciones de riesgo de exclusión social (Jalón y Arias, 2006).

Finalmente, en lo que a la prevención se refiere, la mayoría de los estudios que tratan la reproducción intergeneracional del riesgo, es necesario realizar una intervención multidisciplinar: mejorar las pautas educativas, la calidad de vida de familias de bajo nivel socioeconómico y cultural y ayudando a los niños y niñas desde las primeras etapas de educación (Jalón y Arias, 2006; Serbin y Karp, 2004).

\section{REFERENCIAS BIBLIOGRÁFICAS}

Ainsworth, M. (1993). Some considerations regarding theory and assessment relevant to attachments beyond infancy. En M. Greenberg, D. Cichetti y M. Cummings (eds.): Attachment in the preschool years (pp. 463-488). Chicago: The University of Chicago Press.

Barajas, M.C. y Clemente, R.A. (1991). Ambiente familiar y desarrollo evolutivo. La evaluación de la atención en el hogar. En I Congreso Internacional Infancia y Sociedad. Bienestar y Derechos Sociales de la Infancia. Madrid, Noviembre 1989. Ministerio de Asuntos Sociales

Bassok, D. (2010). Do Black and Hispanic children benefit more from preschool? Understanding differences in preschool effects across racial groups. Child development, 81(6), 1828-1845.

Becker, B. (2011). Social disparities in children's vocabulary in early childhood. does preschool education help to close the gap. The British Journal of Sociology, 62(1), 69-88.

MC Cartney, K., Dearing, E., Taylor, B.A y Bub, K.L.(2007): Quality child care supports the achievement of low-income children: direct and indirect pathways through caregiving and the home environment, Journal of Applied Developmental Psychology, 28(5-6), 411-426. 
Bornstein, M. H., Hahn, C.-S., \& Haynes,0. M. (2011). Maternal personality, parenting cognitions, and parenting practices. Developmental Psychology, 47(3), 658-675.

Bowlby, J. (1980). Attachment and loss: Loss. Basic Books.

Bradley, R. H., \& Corwyn, R. F. (2002). Socioeconomic status and child development. Annual review of psychology, 53(1), 371-399.

Briggs-Gowan, M. J., Carter, A. S., Bosson-Heenan, J., Guyer, A. E., \& Horwitz, S. M. (2006). Are infant-toddler social-emotional and behavioral problems transient?. Journal of the American Academy of Child \& Adolescent Psychiatry, 45(7), 849-858.

Brooks-Gunn, J., \& Duncan, G. J. (1997). The effects of poverty on children. The future of children, 55-71.

Carlson, E., Sroufe, A. y Egeland, B. (2004). The construction of experience: a longitudinal study of representation and behavior. Child Development, 75, 66-83.

Clemente, G. (2011). La atención temprana en la etapa de educación infantil. Universidad de Almería

Conger, R. D., \& Donnellan, M. B. (2007). An Interactionist Perspective on the Socioeconomic Context of Human Development. Annual Review of Psychology., 58(1), 175-199. doi:10.1146/annurev.psych.58.110405.085551

Crittenden, P. M. (1992). Quality of attachment in the preschool years. Development and psychopathology, 4(2), 209-241.

Davis, E., Sawyer, M. G., Lo, S. K., Priest, N., \& Wake, M. (2010). Socioeconomic risk factors for mental health problems in 4-5-year-old children: Australian population study. Academic Pediatrics, 10(1), 41-47.

Defensor del Pueblo. (2007). Violencia escolar: el maltrato entre iguales en la Educación Secundaria Obligatoria 1999-2006. Informes, Estudios y Documentos: Madrid. Recuperado de http://Www.oei.es/oeivirt/Informeviolencia. pdf

Díaz-Aguado Jalón, M. J., \& Martínez Arias, R. (2006). La reproducción intergeneracional de la exclusión social y su detección desde la educación infantil. Psicothema, 18(3).

Elberling, H., Linneberg, A., Olsen, E. M., Goodman, R., \& Skovgaard, A. M. (2010). The prevalence of SDQ-measured mental health problems at age 5-7 years and identification of predictors from birth to preschool age in a Danish birth cohort: The Copenhagen Child Cohort 2000. European child \& adolescent psychiatry, 19(9), 725-735.

Esping-Andersen, G. (2008). Childhood investments and skill formation. International Tax and Public Finance, 15(1), 19-44.

Fajardo Bullón, F., León del Barco, B., Felipe Castaño, E., Polo del Río, M. I., \& Ribeiro Dos Santos, E. J. (2015). Salud mental en menores españoles. Variables socioeducativas. Salud Mental, 38(5), 329-335. doi:10.17711/sm.0185-3325.2015.045

Felfe, C., \& Lalive, R. (2012). child care and child development. What works for whom?. In ceSifo economic Studies and Uc/S conference.

Hartas, D. (2011). The ecology of young children's behaviour and social competence: child characteristics, socio-economic factors and parenting. Oxford Review of Education, 37(6), 763-783. doi:10.1080/03054985.2011.636226

Heckman, J.J. (2006). Skill formation and the economics of investing in disadvantaged children. Science, 312, 1.900-1.902.

Lansford, J. E., Bornstein, M. H., Dodge, K. A., Skinner, A. T., Putnick, D. L., \& Deater-Deckard, K. (2011). Attributions and attitudes of mothers and fathers in the United States. Parenting: Science and Practice, 11, 199-213.

León, B., Felipe, E., Gómez, T., \& López, V. (2011). Acoso escolar en la comunidad de Extremadura 
vs. informe español del defensor del pueblo (2006).

McLoyd VC. 1998. Socioeconomic disadvantage and child development. Am. Psychol. 53:185-204

McLoyd, V. C., Ceballo, R. y Mangelsdorf, S. (1993). The effects of poverty of children's socioemocional development. En J. Noshpitz et al. (Eds), Handbook of child and adolescent psychiatry. New York: Basic Books.

Poulou, M. S. (2013). Emotional and Behavioural Difficulties in Preschool. Journal of Child and Family Studies 24(2), 225-236. doi:10.1007/s10826-013-9828-9

Rodríguez, MC. (2009) Nuevos cambios familiares: la participación paternal en el cuidado infantil. Estudios de Psicología, 30 (3), 331-343.

Rodríguez Hernández y Barrau Alonso (2012). Trastornos del comportamiento. Pediatría Integral; 16(10), 760-768

Sanders, M. (2002). Parenting interventions and the prevention of serious mental health problems in children. The Medical Journal of Australia, 177, 87-92.

Serbin, L. A., \& Karp, J. (2004). The intergenerational transfer of psychosocial risk: Mediators of vulnerability and resilience. Annu. Rev. Psychol., 55, 333-363.

Sveen, T. H., Berg-Nielsen, T. S., Lydersen, S., \& Wichstrøm, L. (2013). Detecting psychiatric disorders in preschoolers: screening with the strengths and difficulties questionnaire. Journal of the American Academy of Child \& Adolescent Psychiatry, 52(7), 728-736.

Thompson, R. (2000). The legacy of early attachments. Child Development,71, 145-152.

Viguer, P y Serra, E. (1996). Nivel socioeconómico y calidad del entorno familiar en la infancia. Anales de psicología, 12(2), 197-205 
